\title{
Supporting the laminated ferromagnetic pole pieces in a magnetic gear: a structure behaviour analysis from a multibody model
}

\author{
M. Desvaux ${ }^{1}$, B. Multon ${ }^{1}$, H. Ben Ahmed ${ }^{1}$ and S. Sire ${ }^{2}$ \\ ${ }^{1}$ SATIE Laboratory, ENS Rennes, Université Bretagne Loire, CNRS, France, e- \\ mail:melaine.desvaux@ens-rennes.fr, bernard.multon@ens-rennes.fr, \\ hamid.benahmed@ens-rennes.fr \\ ${ }^{2}$ Université de Bretagne Occidentale, FRE CNRS 3744, IRDL, France, e-mail: \\ stephane.sire@univ-brest.fr
}

\begin{abstract}
This paper deals with the structure behaviour of a multi-bar system which maintains pole pieces in a magnetic gear. A simplified model of the system is proposed in order to be integrated in a multi-criteria global optimization for the sizing of a magnetic gear in wind power applications. For this purpose, the reduction of the computation time is taken into account. A geometry of the supporting bar is the defined and a Q bars structure is proposed. The study is based on a 1D model for each bar; variable radial and tangential magneto-mechanical pole pieces loads (generated by permanent magnets rings) are also considered. The resolution of the $6 \mathrm{Q} * 6 \mathrm{Q}$ system permits to determine quickly the evolution of displacements for each bar. An example of a magnetic gear with 151 pole pieces (i.e. 3,9 MW wind turbine) is proposed.
\end{abstract}

Key words: Laminated pole pieces, Magnetic gear, Magneto-mechanical load, Multibody, Support bar.

\section{Introduction}

Mechanical gearboxes, currently used in indirect drive electromechanical conversion chain (Fig.1a), provide a lower capital expenditure and lower masses than
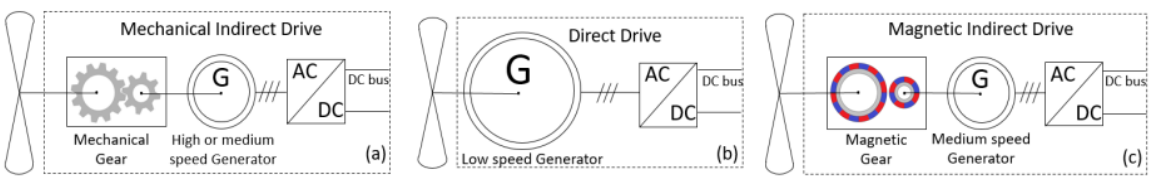

Fig. 1 Wind power conversion chains. (a) mechanical indirect drive, (b) direct drive, and (c) magnetic

indirect drive 
the direct drive conversion chain [1] (Fig.1b). In return, mechanical gearboxes induce production interruptions and repairs, which increase operating costs [2]. In this context, an interesting way is to develop a conversion chain with a mediumspeed generator and a magnetic gear [3] (with non-contact power transmission) (fig.1c). The most studied topology of magnetic gears has been proposed by Martin [4] and was the subject of different behaviour studies proposed by Atallah [5].

This magnetic gear architecture shown in Fig. 2 potentially offers a higher performance with a high torque density and a high reliability than mechanical gearboxes [6] and even more for high torque applications like in high wind turbine (of the order of a few MN.m and a few MW) with only magnetic part consideration [7]. However, no magneto-mechanical sizing has been done on this magnetic gear (with an approach similar than [8] for a wind turbine generator) and it seems to have weaknesses in terms of mechanical strength which are subjected to radial and tangential loads from the magnetic field. Indeed, ferromagnetic pole pieces (see Fig. 3) are very elongated structures, laminated perpendicular to the axis of rotation (to minimize iron losses and conserve a high efficiency of the system) and subjected to magneto-mechanical loads.

The weaknesses of the poles pieces raises the question of the possibility to maintain mechanically this laminated pole pieces without lessening the magnetic properties (therefore without increasing air gaps and modifying magnetic field) in high power wind turbine applications (i.e high dimensions and high pole numbers of the magnetic gear). It is then necessary to evaluate the stiffness of the pole pieces structure for different configurations. This evaluation must be done in a global multi-criteria mechatronic optimization.

The major contribution of this work is the definition of a multibody model of the pole pieces ring that permits to evaluate quickly the displacement generated by the magneto-mechanical variable loads. This multibody model could be integrated in a multi-criteria mechatronic optimization of a magnetic gear for wind turbine

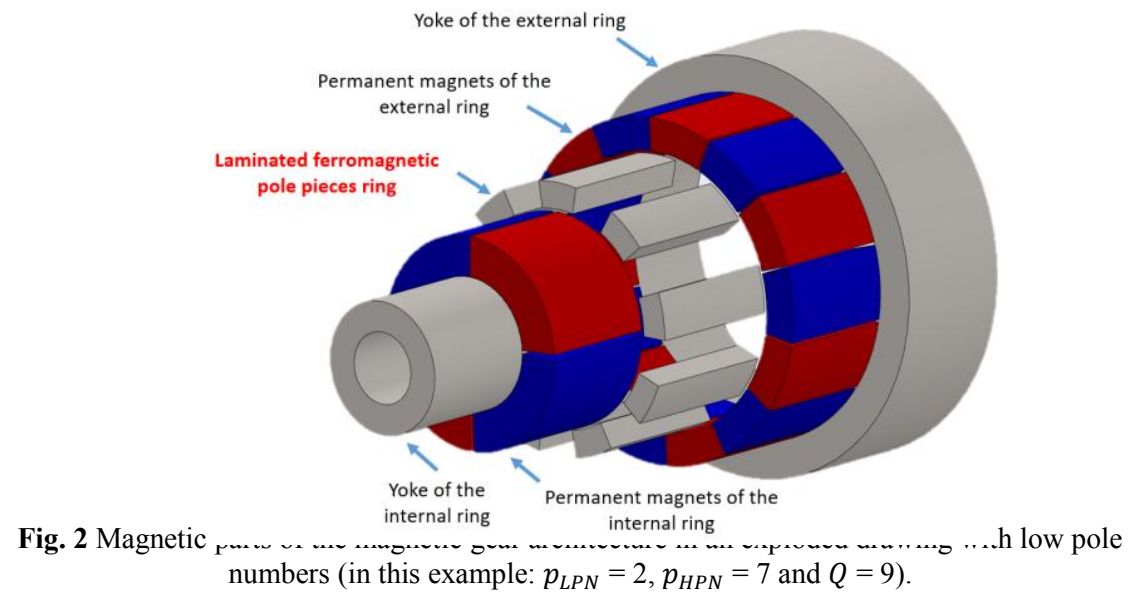


applications.

\section{Support bars for laminated ferromagnetic pole pieces}

\subsection{General principle of the magnetic gear}

The magnetic gear is composed of three rings: a ring with $p_{L P N}$ low pole number of pole pairs of permanent magnets and a ferromagnetic yoke, a ring with $p_{H P N}$ high pole number of pole pairs of permanent magnets and a ferromagnetic yoke, a ring with $\mathrm{Q}$ ferromagnetic pole pieces (an example is given in Fig. 2 with low pole numbers, to improve readability: $p_{L P N}=2, p_{H P N}=7$ and $Q=9$ ).

To achieve the power transmission, three rings pole numbers must respect Eq. (1). Depending on the fixed ring, the gear ratio $G_{\text {magn }}$ is given by Eq. (2) where $\omega_{L P L / 0}, \omega_{H P L / 0}$ and $\omega_{Q / 0}$ are the speed rotation of the internal ring (with low pole numbers), the external ring (with high pole numbers) and the pole pieces ring respectively, $\lambda$ is defined in Eq. (3). For high power applications like offshore wind turbine, the number of pole increases with the diameter. A consequence can be a decrease of their rigidity.

$$
\begin{gathered}
p_{H P N}+p_{L P N}=Q \\
\left\{\begin{array}{c}
\omega_{L P N / 0}=0 \rightarrow G_{m a g n}=\frac{\omega_{H P N / 0}}{\omega_{Q / 0}}=\frac{(\lambda-1)}{\lambda} \\
\omega_{Q / 0}=0 \rightarrow G_{m a g n}=\frac{\omega_{L P N / 0}}{\omega_{H P N / 0}}=\lambda \\
\omega_{H P N / 0}=0 \rightarrow G_{m a g n}=\frac{\omega_{L P N / 0}}{\omega_{Q / 0}}=-(\lambda-1) \\
\lambda=-\frac{p_{H P N}}{p_{L P N}}
\end{array}\right.
\end{gathered}
$$

\subsection{Geometry of support bars}

To support the laminated pole pieces of the magnetic gear, the support bar geometry shown in Fig. 3 is proposed. The support bar is composed of a massive magnetic insulator steel and a magnetic and electrical insulator skin. These support bars do not lessen the magnetic properties; they also do not modify the magnetic field and air gaps. Support bar will then transmit to the structural parts the magneto-mechanical load of the laminated pole pieces. Support bars are 
thus subjected to variable radial and tangential magneto-mechanical loads; their induced displacements and stresses must be then taken into account for their sizing (see [3]).

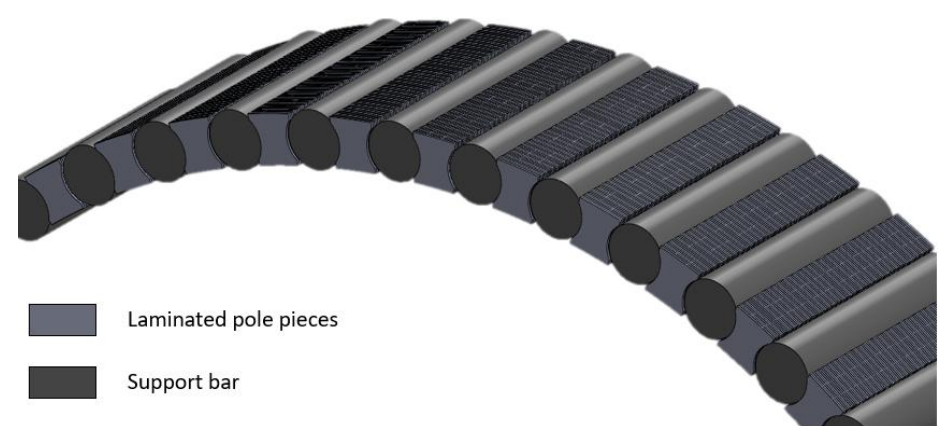

Fig. 3 Pole pieces ring with support bars (end bells are not represented)

\subsection{Magneto-mechanical loads computation}

To compute magneto-mechanical loads, it is possible to take into account exactly the exact geometry of pole pieces with a 2-D magnetic finite element model. This is shown in Fig. 4.a for the magnetic field repartition of a part of the magnetic gear with $p_{L P N}=2, p_{H P N}=7$ and $Q=9$. However, the fact of taking into account the adaptation of the geometry of the laminated pole pieces to be support by the support bars increases strongly the computation time of the magnetomechanical loads determination. Fig. 5 compares the magneto-mechanical loads obtained from a 2-D finite element magnetic model with the adapted geometry of pole pieces (presented in Fig. 4a) and with the theoretical geometry of pole pieces. This theoretical geometry of pole pieces presented in Fig. $4 \mathrm{~b}$ induces a lower computation time of the magneto-mechanical loads since the mesh is simpler. 


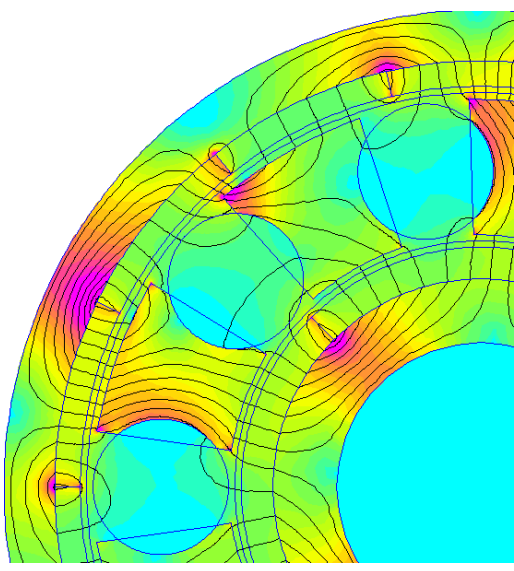

(a)

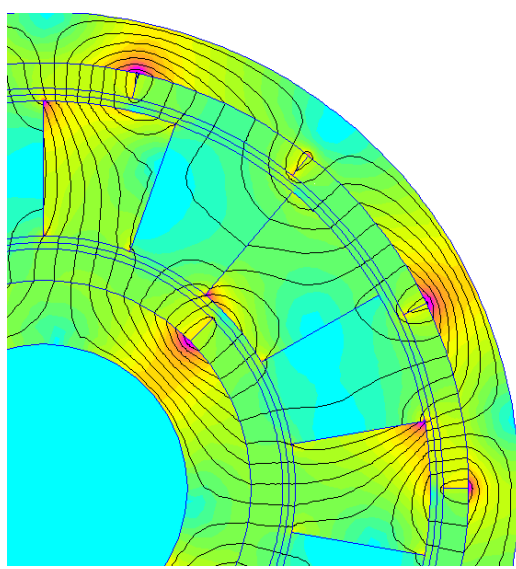

(b)

Fig. 4 Magnetic field repartition obtain from 2-D finite element magnetic model with (a) the adapted geometry of pole pieces and (b) the theoretical geometry of pole pieces for the magnetic gear with $p_{L P N}=2, p_{H P N}=7$ and $Q=9$.

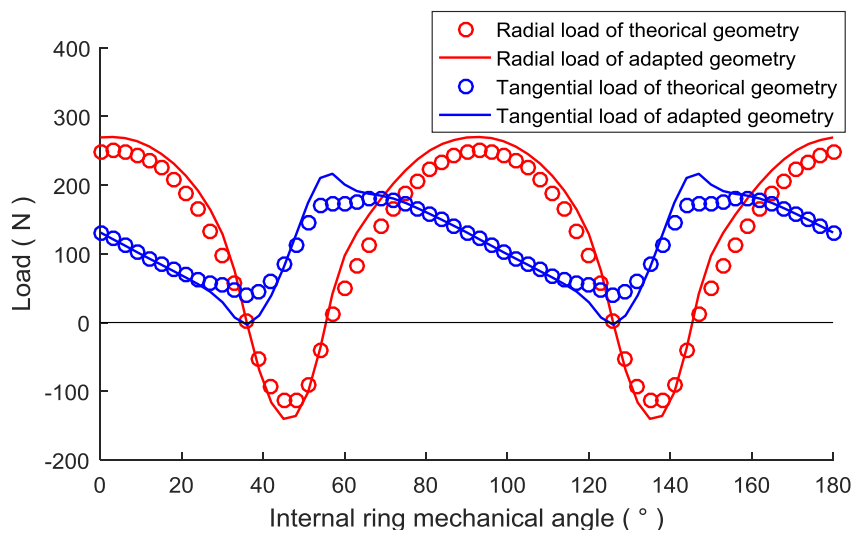

Fig. 5 Comparison between the magneto-mechanical loads obtained from a 2-D finite element magnetic model with the adapted geometry of pole pieces and with the theoretical geometry of pole pieces for a laminated pole piece of the magnetic gear ( a gear with $p_{L P N}=2, p_{H P N}=7$ and $Q=9$ is considered).

The results of the comparison presented in Fig. 5 show that it is possible to compute the magneto-mechanical loads without taking into account to the adapted geometry of the pole pieces. The difference between these two models is indeed lower than $5 \%$. The computation time is then reduced. 


\section{Multibody model of the pole piece ring}

Loads that generate stresses and displacements are the magneto-mechanical radial and tangential loads $\left(\overrightarrow{F_{r}^{(q)}}\right.$ and $\left.\overrightarrow{F_{\alpha}^{(q)}}\right)$ and the weight of the structure for the $Q$ pole pieces and support bars ( $q$ corresponds to the number of the pole pieces, $1 \leq q \leq Q$ ). The geometry of the structural part of the pole pieces ring includes support bars and end bells. It is then possible to consider that a support bar is equivalent to a fixed end beam, considering that the end bells have a negligible deformation compared to support bars. Considering that the magneto-mechanical loads and the weights are applied in the middle of the pole pieces, the pole pieces ring can be modelled with the geometry described in Fig. 6a. Fig. $6 \mathrm{~b}$ defines the multibody model in the middle of the structure.

As shown in Fig. 6b, the multibody model is composed of $4 Q$ bodies $S_{i}^{(q)}$ (with $i=1 . .4$ ) and $4 Q$ degrees of freedom ( $2 Q$ translation and $2 Q$ rotation). The $2 Q$ translations are linked to the stiffness of the support bars and the $2 Q$ rotations correspond to the contacts between pole pieces and support bars. The stiffness of the support bars are determined from a fixed end beam 1-D model. The stiffness of pole pieces are considered negligible in this study because they are laminated.

This multibody model includes $8 \mathrm{Q}$ unknowns for each pole pieces ring. They correspond to the $2 Q$ stiffnesses defined in Eq. (4), the $2 Q$ fixed end reactions at the end of the support bars defined in Eq. (5) and the $4 Q$ reactions from the contact between pole pieces and support bars defined in Eq. (6) as shown in Fig. 6b.

$$
\begin{aligned}
& \left\{\begin{array}{l}
\overrightarrow{T_{x}^{(q)}}=k_{x}^{(q)} \cdot x^{(q)} \cdot \overrightarrow{x_{S, q}} \\
\overrightarrow{T_{y}^{(q)}}=k_{y}^{(q)} \cdot y^{(q)} \cdot \overrightarrow{y_{S, q}}
\end{array}\right. \\
& \left\{\overrightarrow{X_{1}^{(q)}}, \overrightarrow{Y_{2}^{(q)}}\right\} \\
& \left\{\overrightarrow{X_{3}^{(q)}}, \overrightarrow{Y_{3}^{(q)}}, \overrightarrow{X_{4}^{(q)}}, \overrightarrow{Y_{4}^{(q)}}\right\}
\end{aligned}
$$


To determine the $2 Q$ fixed end reaction unknowns, it is possible to consider only $S_{1}^{(q)}$ and determine $\overrightarrow{X_{1}^{(q)}}$ in function of $\overrightarrow{T_{x}^{(q)}}$ from the mechanical equilibrium, and consider only $S_{2}^{(q)}$ and determine $\overrightarrow{Y_{2}^{(q)}}$ in function of $\overrightarrow{T_{y}^{(q)}}$ from the equilibrium to obtain Eq. (7). It remains then $6 Q$ unknowns to be determined. Contrary to the fixed end reaction unknowns, these $6 Q$ unknowns are coupled and must be determined from $6 Q$ coupled equations. Considering $S_{3}^{(q)}$ and then $S_{4}^{(q)}$, it is possible to obtain $6 Q$ coupled relations from Eq. (8) where the two first equations are conditions for equilibrium (which give $4 Q$ equations from projections) and the last equations are equilibrium of torque (which give $2 Q$ equations). The $6 Q$ coupled equations form the matrix system defined in Eq. (9) where $[A]_{6 Q, 6 Q}$ includes projections, $[B]_{6 Q, 1}$ the magneto-mechanical loads and weight and $[X]_{6 Q, 1}$ are the unknowns of the problem.

$$
\left\{\begin{array}{l}
Y_{2}^{(q)}=T_{y}^{(q)} \\
X_{1}^{(q)}=T_{x}^{(q)}
\end{array}\right.
$$
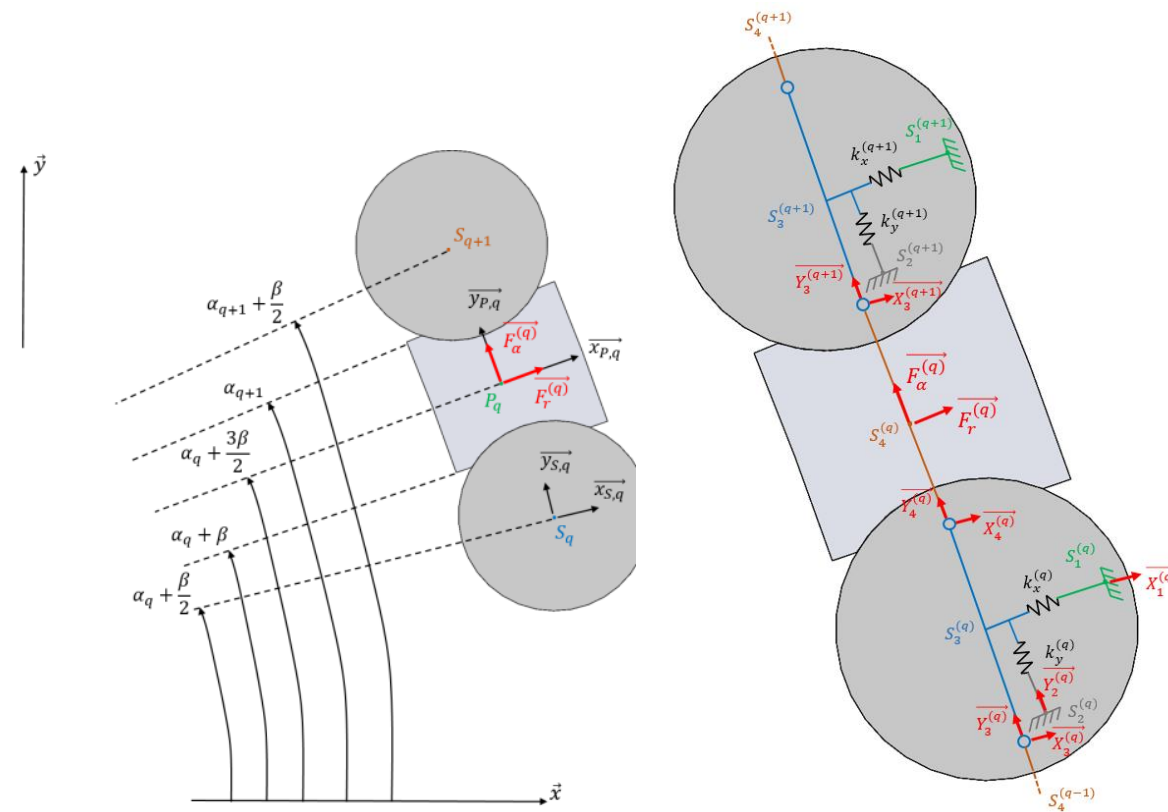

Fig. 6a Multibody modelling of the pole pieces ring Fig. ob Multıbody modelling of the pole with the definition of the different bases pieces ring with the definition of the different loads and bodies. 


$$
\left\{\begin{array}{c}
\overrightarrow{X_{4}^{(q)}}+\overrightarrow{Y_{4}^{(q)}}+\overrightarrow{X_{3}^{(q+1)}}+\overrightarrow{Y_{3}^{(q+1)}}+\overrightarrow{F_{r}^{(q)}}+\overrightarrow{F_{\alpha}^{(q)}}+m_{P}^{(q)} \cdot \vec{g}=\overrightarrow{0} \\
\overrightarrow{X_{4}^{(q)}}+\overrightarrow{Y_{4}^{(q)}}+\overrightarrow{X_{3}^{(q)}}+\overrightarrow{Y_{3}^{(q)}}+\overrightarrow{T_{x}^{(q)}}+\overrightarrow{T_{y}^{(q)}}+m_{S}^{(q)} \cdot \vec{g}=\overrightarrow{0} \\
\left.\overrightarrow{S_{q+1} S_{q}} \wedge \overrightarrow{\left(X_{4}^{(q)}\right.}+\overrightarrow{Y_{4}^{(q)}}\right)+\overrightarrow{S_{q+1} P_{q}} \wedge\left(\overrightarrow{F_{r}^{(q)}}+\overrightarrow{F_{\alpha}^{(q)}}+m_{P}^{(q)} \cdot \vec{g}\right)=\overrightarrow{0} \\
\overrightarrow{S_{q} S_{q+1}} \wedge\left(\overrightarrow{X_{3}^{(q+1)}}+\overrightarrow{Y_{3}^{(q+1)}}\right)+\overrightarrow{S_{q} P_{q}} \wedge\left(\overrightarrow{F_{r}^{(q)}}+\overrightarrow{F_{\alpha}^{(q)}}+m_{P}^{(q)} \cdot \vec{g}\right)=\overrightarrow{0} \\
{[A]_{6 Q, 6 Q} *[X]_{6 Q, 1}=[B]_{6 Q, 1}}
\end{array}\right.
$$

The last $6 Q$ unknowns can be determined from a numerical resolution with an inversion of $[A]_{6 Q, 6 Q}$ which permits to obtain the displacements in the middle of each support bar. Fig. 7a shows the displacements of the support bars generated by the magneto-mechanical loads; Fig. 7b shows the displacements generated by the weight with a coefficient of amplification for the magnetic gear studied in [3]. For this magnetic gear, the maximum calculated radial displacement is $0.5 \mathrm{~mm}$.

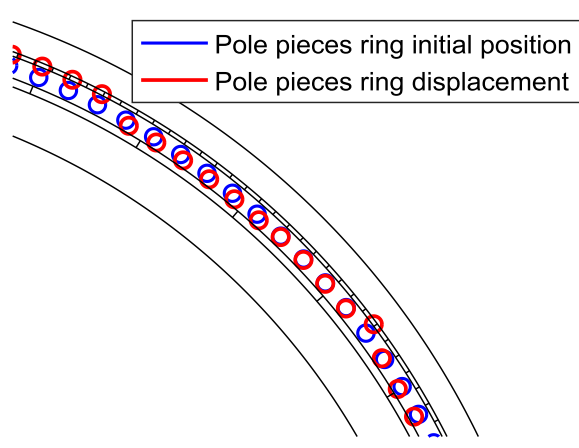

(a)

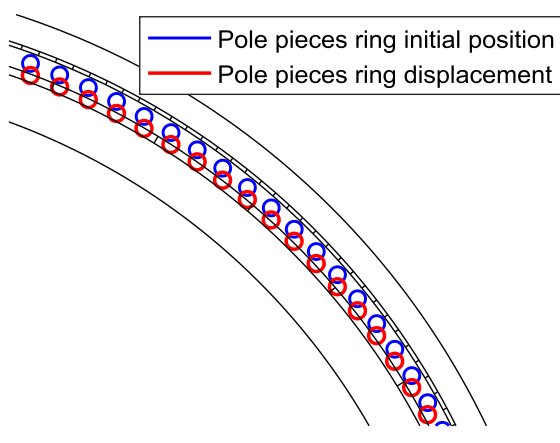

(b)

Fig. 7 Support bars displacements obtained from the multibody model (with a coefficient of amplification) generated by (a) the magneto-mechanical loads and (b) the weight, for the magnetic gear studied in [3].

\section{Loose coupling mechatronic resolution procedure}

The multibody model presented above aims to be integrated in a mechatronic multi-criteria optimization procedure. In this paper, we evaluate support bar displacements considering an acceptable computation time. In order to evaluate the computation time of the problem resolution and to verify if the model is adapted for the mechatronic resolution procedure, an example of the procedure progress is given for the magnetic gear [3] shown in Fig.8. This magnetic gear includes 151 pole pieces. 


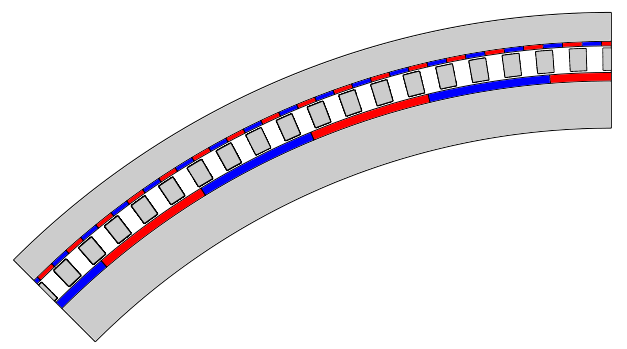

Fig. 8 Magnetic parts of the 3.9 MW and 15 rpm magnetic gear with $p_{L P N}=20, p_{H P L}=131$ and $Q=151$, external diameter $3.8 \mathrm{~m}$ and active length $2 \mathrm{~m}$.

Fig. 9 describes the mechatronic optimization procedure that begins with an initial set of computation parameters. A 2-D magnetostatic computation can then be conducted from a finite element model or an analytical model [9]. This analysis provides the magneto-mechanical loads evolution which includes radial and tangential loads of pole pieces. From this computation, several criteria (objective functions and constraints) can be evaluated like magnetic losses or pole pieces ring displacement (this displacement can be considered as a constraint not to be exceed or an objective function to be minimized). This resolution procedure is then based on the hypothesis of a loose coupling between magneto-mechanical loads and displacement of pole pieces. To complete this optimization procedure, a Particle Swarm Optimization (PSO) algorithm [10] is computed.

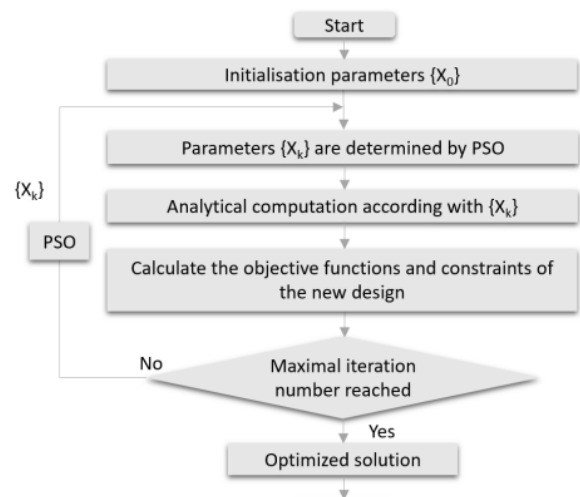

Fig. 9 Mechatronic optimization procedure description [3].

The multibody model described above is then integrated in the mechatronic optimization procedure (block dedicated to the criteria evaluation). For the magnetic 
gear example [9], it is then possible to compute the evolution of the displacement of every support bars of the pole pieces ring. Fig. 10 shows the evolution of the radial and tangential displacements of a support bar due to the magnetomechanical loads and the weight of the structure with a constant rotational speed of $15 \mathrm{rpm}$ for the external ring and $100 \mathrm{rpm}$ for the internal ring.

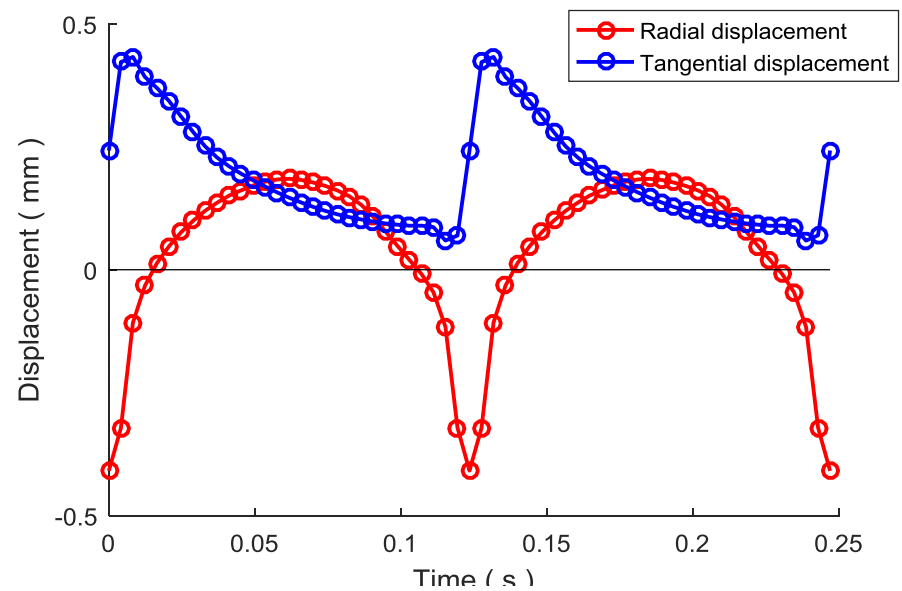

Fig. 10 Evolution of the radial and tangential displacements of a support bar due to the magneto-mechanical loads and the weight for the magnetic gear [3] with a constant rotational speed of $15 \mathrm{rpm}$ for the external ring and $100 \mathrm{rpm}$ for the internal ring.

Fig. 10 shows a periodicity $T$ of the radial a tangential displacements. This periodicity is generated by magneto-mechanical loads and corresponds to the periodicity of the permanent magnetic rings which depends on the rotational speed and the pole pair configuration of the rings as shown in Eq. (10).

$$
T=\frac{2 \pi}{p_{L P N} \cdot \omega_{L P N / 0}}=\frac{2 \pi}{p_{H P N} \cdot \omega_{H P N / 0}}
$$

For the magnetic gear described in [3], the computation time of the displacements for a single position of the different rings is equal to $0.03 \mathrm{sec}$ (with an Intel Xeon E5-1630 v3, 8 threads, $3.70 \mathrm{GHz}$ ) for all of the support bars. If the displacement evolution is computed for 30 positions of the permanent magnet rings, this multibody model then permits to perform the computation in approximately 1 sec. This computation time value is then lower than every finite element mechanical model tested and is acceptable for the 24 hours multi-criteria mechatronic optimization procedure composed of approximately 200 particles and 100 iterations. 


\section{Conclusions}

This paper presents a multibody model developed to evaluate displacements of a multi-bar structure that maintains laminated pole pieces of a magnetic gear. This model aims to be integrated in a multi-criteria mechatronic optimization for the sizing of a magnetic gear for wind power applications. This procedure needs to compute the support bar displacements quickly. The resolution of a $6 Q^{*} 6 \mathrm{Q}$ system permits to determine the evolution of displacements in $1 \mathrm{sec}$ for a magnetic gear with 151 pole pieces (i.e. 3,9 MW magnetic gear adapted for a wind turbine). The displacement evolution computed from the multibody model will permit to evaluate different sizing criteria like stress limit in pole pieces ring or maximal displacement tolerated in airgaps. In prospect, the hypothesis of loose coupling between the magneto-mechanical loads and the displacement of pole pieces must be analyzed to validate the mechatronic resolution procedure.

\section{References}

1. R. Lacal Arántegui and J. Serrano Gonzáles, 2014 JRC wind status report: Technology, market and economic aspects of wind energy in Europe. 2015.

2. J. Keller, Y. Guo, and L. Sethuraman, "Gearbox Reliability Collaborative Investigation of Gearbox Motion and High-Speed-Shaft Loads," NREL, Tech. Rep. TP-5000-65321, 2016.

3. M. Desvaux, \& al., "Design and optimization of magnetic gears with arrangement and mechanical constraints for wind turbine applications," in 2016 11th International Conference on Ecological Vehicles and Renewable Energies, EVER 2016, 2016.

4. T. B. Martin, "Magnetic transmission,” Pat. US3378710, 1968.

5. K. Atallah and D. Howe, “A novel high-performance magnetic gear," IEEE Trans. Magn., vol. 37, no. 4, pp. 2844-2846, 2001

6. E. Gouda, S. Mezani, L. Baghli, and A. Rezzoug, "Comparative study between mechanical and magnetic planetary gears," IEEE Trans. Magn., vol. 47, no. 2, pp. 439-450, 2011.

7. D. Matt, \& al., "Design of a Mean Power Wind Conversion Chain with a Magnetic Speed Multiplier," in Chap. 10 of InTech book "Advances in Wind Power, 2012, pp. 247-266.

8. A. Zavvos, A. S. Mcdonald, and M. Mueller, "Structural optimisation tools for iron cored permanent magnet generators for large direct drive wind turbines," in IET Conference on Renewable Power Generation (RPG 2011), 2011.

9. M. Desvaux, B. Traullé, R. L. G. Latimier, S. Sire, B. Multon, and H. Ben Ahmed, "Computation Time Analysis of the Magnetic Gear Analytical Model," IEEE Trans. Magn., vol. 53, no. 5, pp. 1-10, 2017.

10. S. Niu, and al., "Design optimization of magnetic gears using mesh adjustable finite-element algorithm for improved torque," IEEE Trans. Magn., vol. 48, no. 11, pp. 4156-4159, 2012.

11. J. Aubry, H. Ben Ahmed, and B. Multon, "Sizing optimization methodology of a surface permanent magnet machine-converter system over a torque-speed operating profile: Application to a wave energy converter," IEEE Trans. Ind. Electron., vol. 59, no. 5, pp. 2116-2125, 2012. 